\title{
Electric Dipole Moments From Dark Sectors
}

\section{Shohei Okawa*}

Department of Physics and Astronomy, University of Victoria

E-mail: bkawaduvic.ca

\begin{abstract}
We examine the sensitivity of electric dipole moments (EDMs) as precision observables for new $\mathrm{CP}$-violating physics in a hidden (or dark) sector. Assuming that the dominant mediation channel is via one or more of the vector, Higgs or neutrino portals, we examine the leading EDM contributions. The dominant contributions arise at two-loop order, and EDMs can provide sensitivity to portal couplings that is complementary to direct probes at the intensity frontier or high energy colliders. In particular, we identify a significant two-loop contribution to the electron EDM, mediated through the singlet (Higgs plus neutrino) portal, for which EDMs already provide new and complementary sensitivity in the regime of large singlet neutrino masses.
\end{abstract}

XXIX International Symposium on Lepton Photon Interactions at High Energies - LeptonPhoton2019 August 5-10, 2019

Toronto, Canada

${ }^{*}$ Speaker. 


\section{Introduction}

Recently, significant attention has been paid to models of physics involving hidden (or dark) sectors, as explanations of empirical deficiencies of the Standard Model (SM), such as neutrino mass and dark matter. As a typical characteristic, such physics models may involve new degrees of freedom with a mass well below the electroweak scale. The primary assumption is that all new fields are neutral under SM gauge symmetries, implying in particular that the chiral electroweak $S U(2)_{L} \times U(1)_{Y}$ structure of the SM is maintained. The effective Lagrangian at the electroweak scale then takes the form

$$
\mathscr{L}_{\mathrm{NP}}=\mathscr{L}_{\mathrm{IR}}+\sum_{d \geq 5} \frac{1}{\Lambda_{\mathrm{UV}}^{d-4}} \mathscr{O}_{d},
$$

with new UV physics described by a series of higher dimensional operators $\mathscr{O}_{d}$ constructed from SM fields. Notably, new IR physics contained in $\mathscr{L}_{\mathrm{IR}}$ is highly constrained by this effective field theory (EFT) framework, as it must be UV-complete. The simplest SM-neutral IR hidden sector allows only new scalars $S_{i}$, neutral fermions $N_{i}$ and/or new $U(1)^{\prime}$ gauge boson(s) $A_{\mu}^{\prime}$ to mediate direct interactions with the SM. Indeed, the only renormalizable, and thus UV-complete, interactions (or portals) for these fields with the SM can be written in the form

$$
\mathscr{L}_{\mathrm{IR}}=\varepsilon B^{\mu v} F_{\mu v}^{\prime}-\left(A S+\lambda S^{2}\right) H^{\dagger} H-Y_{N} \bar{L} H N+\mathscr{L}_{\text {hid }},
$$

possibly generalized to include multiple copies of these mediator fields, e.g. a complex extension of $S$ charged under $U(1)^{\prime}$ etc . Once coupled to the SM through these channels, the IR hidden sector described by $\mathscr{L}_{\text {hid }}$ can be almost arbitrarily complicated.

Dark sector models are often best probed using high-precision low energy observables. As a prime example, electric dipole moments (EDMs) of atoms, molecules and nucleons have for many years provided important indirect constraints on $C P$-violating new physics at or above the electroweak scale [ $\mathrm{⿴}]$. In this work, motivated by recent progress in the measurement of the electron $\operatorname{EDM} \mathscr{L}=-\frac{i}{2} d_{e} \bar{e} \sigma^{\mu v} \gamma_{5} e F_{\mu v}$ at the $\operatorname{ACME}\left(\left|d_{e}\right| \leq 1.1 \times 10^{-29} e \mathrm{~cm}\right)[[]]$, we revisit the generation of the EDM from dark sectors. The impact on the EDM of adding dark sector degrees of freedom coupled through the scalar, vector and neutrino portals has been studied for some time in the literature [3, 田, []. It was found, however, that the contributions to the electron EDM are generically suppressed well below the level of current sensitivity, $\left|d_{e}\right|<10^{-33} \mathrm{ecm}$ [䧃], due in part to the small neutrino mass scale or strong limits on the gauge kinetic mixing $\varepsilon$. Here, we identify a new mediation channel, which we term the singlet portal, involving both the neutrino and scalar portals, which can induce a sizeable electron EDM. The singlet portal allows EDM contributions which avoid the primary suppression factors noted above. We will make the assumption that $C P$ phases in the hidden sector are maximal, and determine the scale of the induced EDMs, given the restrictions already in place on the portal couplings from a variety of other experimental probes.

\section{EDM contributions analyzed so far}

In this section, for comparison with a new contribution in Section 3, we will present two (suppressed) contributions to EDMs from a generic dark sector, coupled to the SM only via the three portal interactions. ${ }^{1}$

\footnotetext{
${ }^{1}$ For more comprehensive study, see [四].
} 


\subsection{Neutrino portal}

The neutrino portal $\bar{L} H N$ allows for $C P$-violation in the portal interaction $Y_{N}$ itself. As the simplest seesaw model for neutrino mass generation, and for leptogenesis, the neutrino portal has been studied extensively, including its contribution to lepton EDMs. In order to incorporate a nontrivial $C P$ phase, we require two singlet fermions $N_{R}, N_{S}$ leading to the following mass matrix for $\left(v_{L}, N_{R}, N_{S}\right)$,

$$
\mathscr{M}=\left(\begin{array}{ccc}
0 & m_{D_{1}} & m_{D_{2}} \\
m_{D_{1}} & M_{R} & \Delta M \\
m_{D_{2}} & \Delta M & M_{S}
\end{array}\right),
$$

where $m_{D_{i}}$ are the Dirac masses and we work in the regime $M_{R, S} \gg m_{D_{i}}, \Delta M$, where the mass spectrum includes a light neutrino $m_{v} \simeq\left(m_{D_{1}}^{2}-m_{D_{2}}^{2}\right) / M$, and heavy states $M_{+}^{0} \simeq M_{S}, M_{-}^{0} \simeq M_{R}$, with $M=\left(M_{R}+M_{S}\right) / 2$ the Majorana mass scale. As shown in Fig. 1 (left), an EDM is generated at two loop order [四, 四],

$$
d_{e} \sim\left(3 \cdot 10^{-35} e \mathrm{~cm}\right) \frac{m_{D_{1}}^{2} m_{D_{2}}^{2}}{M^{4}} \frac{M_{S}^{2}-M_{R}^{2}}{\mathrm{GeV}^{2}} .
$$

The ratios $\theta_{v} \sim m_{D_{i}} / M \lesssim 10^{-1}$ are the visible-hidden mixing angles and, even with considerable tuning, the constraints on the light neutrino mass spectrum limit the EDM to less than $10^{-33} \mathrm{e} \cdot \mathrm{cm}$, well below the current experimental limit.

\subsection{Dark Barr-Zee mechanism}

As noted in [\$]], combining the scalar and vector portals allows the generation at 2-loop order of the 'dark EDM' of a SM fermion such as the electron,

$$
d_{e}^{\text {dark }} \bar{e} \sigma^{\mu v} i \gamma_{5} e F_{\mu v}^{\prime} .
$$

Since $A_{\mu}^{\prime}$ is generically massive, integrating it out in the latter case generates a higher-dimension 'EDM radius' (or Schiff moment), rather than an EDM,

$$
\bar{e} \sigma^{\mu v} i \gamma_{5} e F_{\mu v}^{\prime} \longrightarrow \frac{1}{m_{A^{\prime}}^{2}} \bar{e} \sigma^{\mu v} i \gamma_{5} e \square F_{\mu v}
$$

As an explicit example to generate the dark EDM operator, a generic Barr-Zee-type contribution is shown in Fig. 1 (middle) [5]. This involves the $C P$-odd coupling of the scalar $S$ to a dark sector fermion $\psi, \bar{\psi}\left(m_{\psi}+S\left(Y_{S}+i \tilde{Y}_{S} \gamma_{5}\right)\right) \psi$, which is in turn charged under $A_{\mu}^{\prime}$. Integrating out $\psi$ leads to a $C P$-odd $S F^{\prime} \tilde{F}^{\prime}$ operator, which in turn can generate the dark EDM of an electron. The contributing diagrams were analyzed in [5] and, with the hierarchy $m_{A^{\prime}} \ll m_{S} \ll m_{\psi}$ and Higgs-scalar mixing angle $\theta_{h} \ll 1$, one finds the EDM radius,

$$
r_{d_{e}}^{2}=\frac{|e| \alpha^{\prime} \tilde{Y}_{S} m_{e}}{16 \pi^{3} v m_{\psi} m_{A^{\prime}}^{2}} \times \varepsilon^{2} \theta_{h} \ln \left(m_{\psi}^{2} / m_{S}^{2}\right) .
$$

An estimate of the resulting EDM probed in atomic/molecular EDM experiments, induced by the mixing of $s-p$ orbitals, follows by identifying the corresponding scale with the $K$-shell radius, $d_{e} \sim\left(Z \alpha m_{e}\right)^{2} r_{d_{e}}^{2}$, given $m_{A^{\prime}}>Z \alpha m_{e}$. This leads to the estimate [[]]

$$
d_{e} \sim\left(4 \cdot 10^{-33} e \cdot \mathrm{cm}\right) \times\left(\frac{1 \mathrm{GeV}}{m_{\psi}}\right)\left(\frac{\varepsilon}{10^{-4}}\right)^{2}\left(\frac{\theta_{h}}{10^{-3}}\right)
$$



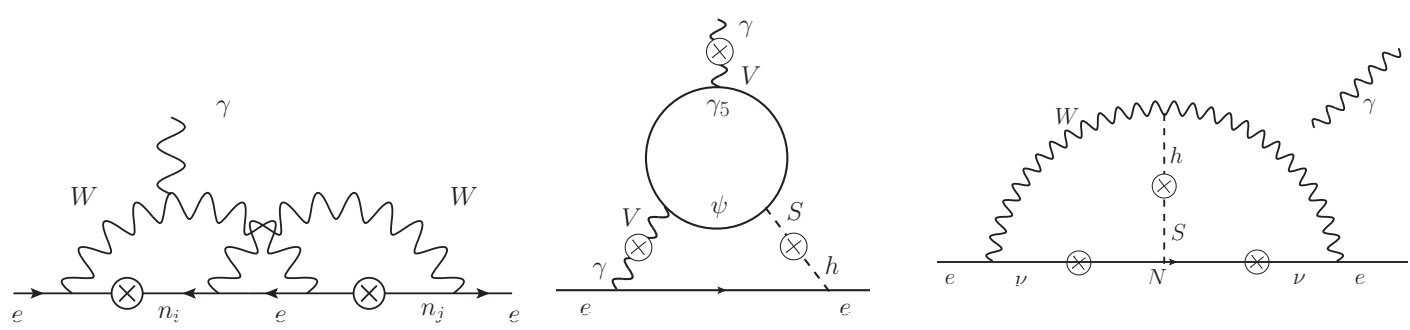

Figure 1: 2-loop contributions to lepton EDMs from dark sector models: (left) an example of the leading 2-loop contribution due to $C P$-violation in the neutrino portal; (middle) a 2-loop contribution induced by the dark EDM of the electron, mediated by the vector and scalar portals; and (right) a new 2-loop contribution mediated by the combined singlet portal. The crosses denote portal couplings.

which is still well below the current sensitivity to the electron EDM, due in part to the strong limit on $\varepsilon$ in the relevant $A_{\mu}^{\prime}$ mass range from $g-2$ of the electron and direct searches for dark photon at NA64 and BaBar.

\section{EDMs from the singlet portal}

In this section we shall focus on a different channel which appears to provide the largest EDM contribution, given current constraints on the portal mixing angles. If we combine the neutrino and singlet scalar portals,

$$
\mathscr{L}_{\text {singlet }}=-A S H^{\dagger} H-\left(Y_{N} \bar{L} H N+\text { h.c. }\right),
$$

a combination that we term the singlet portal, a new 2-loop contribution to EDMs can be identified as shown in Fig. 1 (right). We have introduced a further pseudoscalar dark sector coupling $\lambda_{N} S \bar{N} i \gamma_{5} N$ between $S$ and $N$, which therefore breaks $C P$ in the full theory and allows for EDMs at 2-loop order. This mediation channel does not require any additional dark sector degrees of freedom, or multiple generations of fermions. The addition of the scalar portal allows a number of the

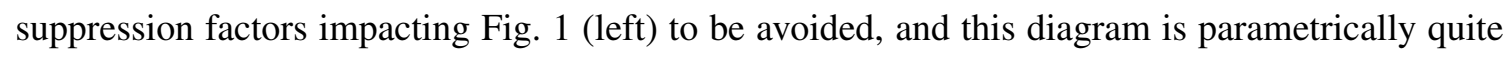
large, given the limited constraints on the neutrino and scalar mixing angles. Most importantly, $N$ can be chosen to be a Dirac particle, and thus unconstrained by the visible neutrino mass splitting.

In this section, we will show the size of the new 2-loop EDM contribution, and explore the complementarity of the EDM sensitivity to other direct collider and fixed target probes. Details of

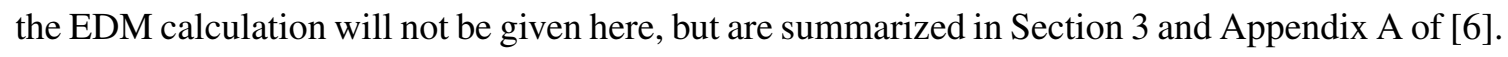
The calculation can be performed at leading order in the weak interactions $\left(\alpha_{W} \rightarrow 0\right)$, utilizing only the Goldstone components $G^{ \pm}$of the weak vector bosons. After electroweak symmetry breaking, the relevant portal couplings take the form,

$$
\mathscr{L}_{\text {singlet }}=\frac{\theta_{h}}{v}\left(m_{S}^{2}-m_{h}^{2}\right) S\left(v h+G^{+} G^{-}\right)+\frac{\sqrt{2} \theta_{v}}{v}\left[G^{-} \bar{e}\left(m_{e} P_{L}+m_{N} P_{R}\right) N+h . c .\right]+\cdots
$$

where $\theta_{h}=A v /\left(m_{S}^{2}-m_{h}^{2}\right)$ is the scalar mixing angle, while $\theta_{v}$ is the corresponding singlet neutrino

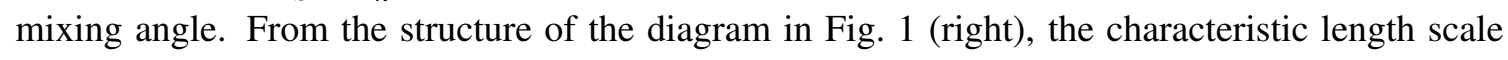
$L_{e}^{\text {scale }}$ of the 2-loop contribution to $d_{e}$ takes the form,

$$
L_{e}^{\text {scale }}=\frac{\lambda_{N} \theta_{v}^{2} \theta_{h}}{\left(16 \pi^{2}\right)^{2}} \times \frac{2 m_{e} m_{N}}{v^{3}} \sim 4 \times 10^{-27} \mathrm{~cm} \times \lambda_{N} \theta_{v}^{2} \theta_{h} \times \frac{m_{N}}{m_{W}} .
$$




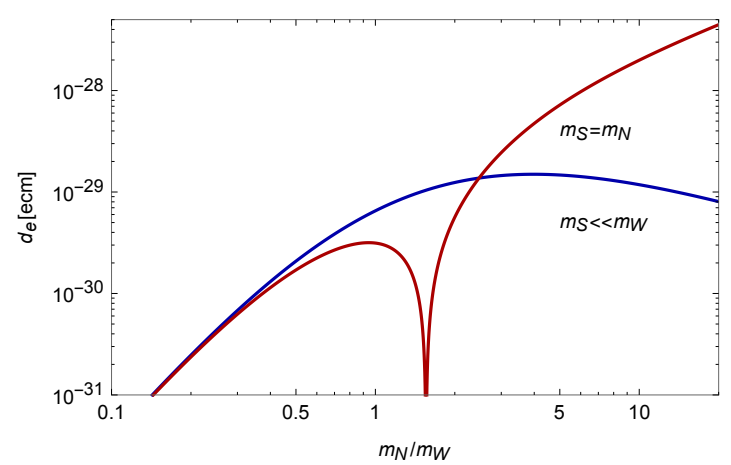

Figure 2: For the singlet portal example, the size of the electron EDM is shown as a function of $m_{N} / m_{W}$ for two different choices of the singlet scalar mass: $m_{S} \ll m_{W}$ and $m_{S}=m_{N}$. We have set $\theta_{h} \theta_{v}^{2}=10^{-2}$.

After a lengthy calculation, the corresponding amplitude can be written in terms of the overall length scale (B]) and the loop momenta $q$ and $k$,

$$
\mathscr{M}=-\frac{i}{2} e L_{e}^{\text {scale }} \bar{e} \sigma^{\mu v} \gamma_{5} e F_{\mu v} \times \int d^{4} q d^{4} k f_{\text {scalar }}(q, k),
$$

so that

$$
d_{e}=e L_{e}^{\text {scale }} \times \int d^{4} q d^{4} k f_{\text {scalar }}(q, k),
$$

where $f_{\text {scalar }}$ is a dimensionless scalar integrand. Numerical results in two scaling regimes for $m_{S}$ are shown in Fig. $\square$. The resonant behaviour for $m_{S} \rightarrow m_{h}$ is apparent in the case that $m_{S}=m_{N}$, with the suppression of the EDM due to the fact that $\theta_{h}=A v /\left(m_{S}^{2}-m_{h}^{2}\right)$ is held fixed. The relatively weak decoupling as $m_{N} \rightarrow \infty$ is reminiscent of the dependence of many FCNC observables on $m_{t}$.

To exhibit a characteristic scale for the EDM, in the mass range $m_{N} \sim(1-10) m_{W}$, with $m_{S} \ll m_{W}$, we find numerically

$$
d_{e} \sim 10^{-29} e \mathrm{~cm} \times\left(\frac{\lambda_{N} \theta_{v}^{2} \theta_{h}}{10^{-2}}\right) .
$$

This is quite close to the current experimental limit from ACME of $1.1 \times 10^{-29} \mathrm{ecm}$, assuming relatively mild constraints on the mixing angles, and is the largest EDM contribution we have uncovered according to our definition of a UV-complete dark sector. The full scaling of the EDM with $m_{N}$ and $m_{S}$ is illustrated in Fig. B for two different portal mass regimes, where we also exhibit the leading direct constraints on the mixing angles for comparison. The constraints on the neutrino mixing angle are from a CHARM search for heavy neutral leptons, DELPHI searches for $Z \rightarrow N v$, an ALEPH measurement of $W$-pair production, and precision electroweak data (EWPD) including lepton universality. The relevant limits in each mass range are combined with limits on Higgs-scalar mixing from an L3 search for $e^{+} e^{-} \rightarrow Z^{*} S$, to produce the limit contours in Fig. B. For $m_{S} \ll m_{W}$, the limit on Higgs mixing is $\theta_{h} \sim 0.1$, while it weakens to $\mathscr{O}(1)$ for larger $m_{S}$. This results in the slightly weaker direct limits shown in Fig. [3 (right) for larger masses. Note that the stringent limits on lepton flavour violating processes cannot be directly applied to $\theta_{v}$ without further assumptions about the flavour structure of the $N$-sector. 

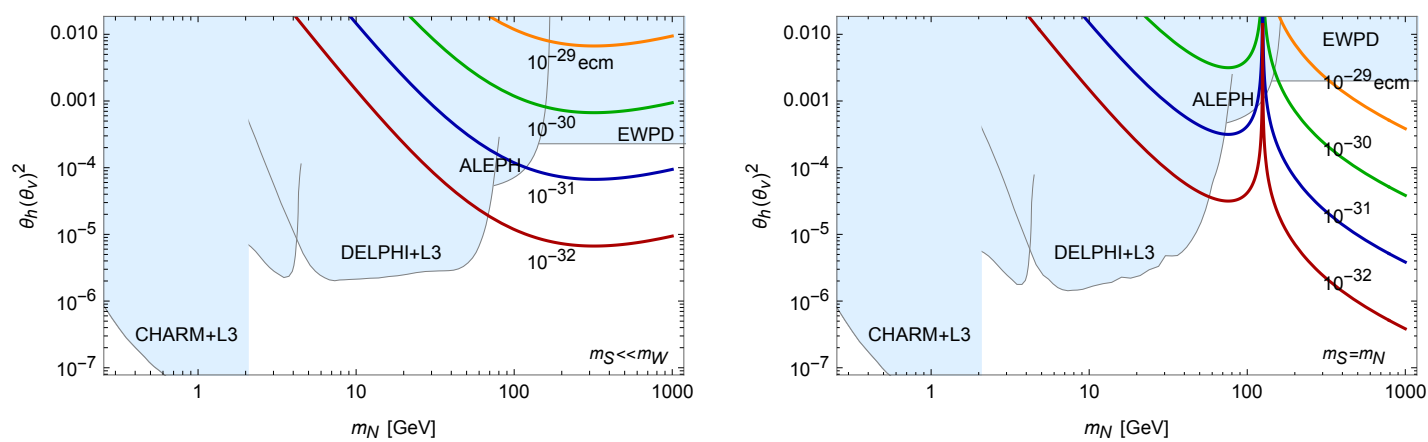

Figure 3: For the singlet portal example, EDM sensitivity contours (with a maximal $C P$ phase) are shown as a function of the singlet neutrino mass, assuming (left) $m_{S} \ll m_{W}$ and (right) $m_{S}=m_{N}$. Note that the current limit from the ACME collaboration is $1.1 \times 10^{-29} \mathrm{ecm}$. For comparison, the limits on the portal couplings are shown in light blue. See the text for further details.

\section{Conclusions}

The majority of the EDM contributions from dark sector $C P$-violation are below current experimental sensitivity, primarily due to the significant constraints on the portal couplings from independent measurements. Nonetheless, we have identified contributions from the singlet $N-S$ portal that can be sizeable, and it is apparent that EDMs can provide sensitivity to the portal mixing angles that is complementary for large $m_{N}$ due to the mild decoupling behaviour. Moreover, the current limit on the electron EDM from the ACME experiment already provides sensitivity to this model that is comparable in reach to collider probes. Should the next round of improvements (by ACME and/or other collaborations searching for $d_{e}$ ) lead to a positive detection, one would not be able to unambiguously assign it to models of new physics with charged particles. It could also be a signature of neutral dark sectors near the weak scale. For models with electroweak scale singlet fermions $N$, the upcoming high-luminosity run of the LHC may provide the best probe.

\section{References}

[1] M. Pospelov and A. Ritz, Electric dipole moments as probes of new physics, Annals Phys. 318 (2005) 119-169, [hep-ph/0504231].

[2] ACME Collaboration, V. Andreev et al., Improved limit on the electric dipole moment of the electron, Nature 562 (2018), no. 7727 355-360.

[3] D. Ng and J. N. Ng, A Note on Majorana neutrinos, leptonic CKM and electron electric dipole moment, Mod. Phys. Lett. A11 (1996) 211-216, [hep-ph/9510306].

[4] J. P. Archambault, A. Czarnecki, and M. Pospelov, Electric dipole moments of leptons in the presence of majorana neutrinos, Phys.Rev. D70 (2004) 073006, [hep-ph/0406089].

[5] M. Le Dall, M. Pospelov, and A. Ritz, Sensitivity to light weakly-coupled new physics at the precision frontier, Phys. Rev. D92 (2015), no. 1 016010, [arXiv:1505.01865]].

[6] S. Okawa, M. Pospelov, and A. Ritz, Electric Dipole Moments From Dark Sectors, arXiv:1905.05219. 